\title{
The Nature of Interchain Excitations in Conjugated Polymers: Spatially-Varying Interfacial Solvatochromism of Annealed MEH-PPV Films Studied by Near-Field Scanning Optical Microscopy (NSOM)
}

\author{
Richard D. Schaller, Lynn F. Lee, Justin C. Johnson, Louis H. Haber, and \\ Richard J. Saykally* \\ Department of Chemistry, University of California at Berkeley, Berkeley, California 94720-1460 \\ John Vieceli and Ilan Benjamin* \\ Department of Chemistry, University of California, Santa Cruz, Santa Cruz, California 95064

\begin{abstract}
Thuc-Quyen Nguyen ${ }^{\dagger}$ and Benjamin J. Schwartz*
Department of Chemistry and Biochemistry, University of California, Los Angeles, Los Angeles, California 90095-1569
\end{abstract}

Received: December 19, 2001; In Final Form: June 3, 2002

\begin{abstract}
The nature of interchain electronic species in conjugated polymers has been the subject of much debate. In this paper, we exploit a novel near-field scanning optical microscopy (NSOM)-based solvatochromism method to spatially image the difference in dipole moment, and hence the difference in degree of charge separation, between the ground and electronic excited states of the emissive interchain species in films of poly(2-methoxy5-(2'-ethylhexyloxy)-1,4-phenylene vinylene) (MEH-PPV). The method uses NSOM to collect emission from near the surface of solid samples that are placed into contact with liquids of varying polarity. The solvatochromic spectral shifts of the interfacial luminescence are measured as a function of solvent polarity; the results are analyzed with an interfacial dielectric continuum model to determine the dipole moment of emissive excited states. Experiments performed on films of the laser dye trans-4-dicyanomethylene-2-methyl-6-p-dimethylaminostyryl-4H-pyran (DCM) in poly(methyl methacrylate) (PMMA) demonstrate that our interfacial NSOM solvatochromic method and analysis can successfully reproduce the known dipole change of DCM upon photoexcitation. With the method calibrated, we then apply it to the interchain luminescence from the surface of thermally annealed MEH-PPV films. The interfacial solvatochromic analysis reveals that the dominant interchain species in annealed MEH-PPV films is "excimer-like", exhibiting an 4-7 D decrease in dipole moment upon optical excitation. In a few highly localized regions of the film (ca. 1-2 $\mu \mathrm{m}$ in diameter), however, the interchain excited state exhibits a large $(\sim 9-13 \mathrm{D})$ increase in dipole moment upon excitation, indicative of minority interchain species with a large degree of charge separation, such as exciplexes or polaron pairs. The large variation in excited-state dipole moments observed throughout the film is suggestive of an entire family of interchain species, each characterized by a different degree of charge separation. The fact that the large-dipole interchain species are found in spatially segregated domains implies that interchain charge separation in conjugated polymer films is associated with the presence of defects. When the molecular weight of the polymer is lowered, the large excited-state dipole regions increase in spatial extent, suggesting that the defects that promote charge separation are intrinsic and may be associated with the chain ends.
\end{abstract}

\section{Introduction}

Conjugated polymers have attracted great attention during the past decade due to their potential for use in optoelectronic devices. ${ }^{1-3}$ Despite the myriad of applications and correspondingly large number of photophysical studies on these materials, there remains controversy about the significance and nature of interchain electronic species in conjugated polymer films. Estimates of the fraction of primary photoexcitations that result in interchain species range from essentially zero $^{4,5}$ to over

* To whom correspondence should be addressed. E-mail addresses: saykally@uclink4.berkeley.edu; benjamin@chemistry.ucsc.edu; schwartz@ chem.ucla.edu.

Current address: Department of Chemistry, Columbia University, New York, New York 10027.
$90 \% .^{6-8}$ The presence of interchain electronic species has important implications for the performance of devices based on these materials: interchain species may be responsible for quenching a conjugated polymer's luminescence but also may be beneficial for promoting charge transport. ${ }^{9-11}$ There is also debate concerning the physical nature of interchain electronic species, which are often referred to in the literature as "excimers", 8,12-14 "aggregrates", 9,15-20 or "polaron pairs". ${ }^{6-8,21-23}$ The way that we distinguish among these labels is to use "excimer" to denote a neutral excitation shared equally between two or more chromophores in the electronic excited state and "aggregate" to imply delocalization of the wave function over multiple chain segments in both the ground and excited states. We reserve use of "polaron pair" for interchain excited states 
characterized by charge separation, such as states with an electron residing on one chain segment that is Coulombically bound to a hole on a neighboring chain segment. We refer to interchain excited states that undergo only partial charge separation as "exciplexes".

In previous work, the UCLA group has argued that the controversy concerning interchain species in conjugated polymers stems from the fact that the formation of interchain species depends critically on the way the polymer chains pack in the film. ${ }^{9}$ The chain-packing morphology, in turn, is sensitive to the history of how the film was prepared; important factors include the way the polymer is dissolved into solution, ${ }^{24}$ the spin speed, ${ }^{25}$ and the choice of solvent and concentration of the polymer solution from which the film is cast. ${ }^{9,10}$ Because different groups tend to prepare their films in different ways, it is not surprising that there has been such widespread disagreement concerning the results of both photophysics and device experiments. Part of the controversy also may result from the fact that interchain (Förster) energy transport in conjugated polymers is quite facile, ${ }^{26}$ so single-chain excitations are able to rapidly migrate to "defect" sites to produce interchain species. Given that there are an essentially infinite number of ways that the chains can pack, one might expect a conjugated polymer film to contain a variety of interchain excited states, blurring the distinction between the commonly applied interchain labels.

Most of what is known concerning the presence of interchain species in conjugated polymer films comes from a combination of steady-state and time-resolved spectroscopic measurements. The primary photophysical signature of interchain species is the presence of a weak, red-shifted emission that is longer-lived than the single-chain exciton emission observed from dilute conjugated polymer solutions. ${ }^{9,12-14,17}$ This weak emission could result from either aggregates or excimers, both of which are expected to be lower in energy than the single-chain exciton and have long radiative lifetimes; ${ }^{27}$ the red-shifted emission might also result from the thermally activated recombination of polaron pairs. ${ }^{6,8,21}$ In films of some conjugated polymers, the presence of a weak absorption band that is red-shifted from that of a single chain provides evidence that interchain species (aggregates) also can form in the ground electronic state..$^{9,16,18,28}$ While all of these studies have provided some understanding regarding the dynamics of interchain species and their effects on luminescence quantum yields, there is still relatively little known concerning the electronic nature of the intermolecular excited state, and which of the various interchain labels, if any, is most appropriate.

In this paper, we seek to elucidate the nature of interchain interactions in conjugated polymer films by exploiting solvatochromism. Photoluminescence (PL) solvatochromism is a powerful method for investigating the electronic properties of excited states by systematically changing the dielectric properties of the surrounding environment. ${ }^{29,30}$ For most chromophores, the ground- and excited-state dipoles are parallel, an assumption that we will make throughout this work. ${ }^{31}$ For parallel dipole moments, if the excited-state dipole is smaller than that of the ground state, as expected for an excimer, then increasing the polarity of the environment will stabilize the ground state more than the excited state, resulting in a blue shift of the emission. Conversely, if the excited-state dipole is larger than that of the ground state, as would be the case for an interchain polaron pair, then increasing the polarity of the solvent will stabilize the excited state more than the ground state, producing a redshifted emission. Solvatochromic measurements can determine quantitatively the difference between the ground- and excited- state dipole moments $\left(\Delta \mu_{\mathrm{e}}\right)$ from the spectral shift of the emission band maximum $(\bar{v})$ as a function of the static dielectric constant $\left(\epsilon_{\mathrm{s}}\right)$ and refractive index $(n)$ of the surrounding environment through the Lippert-Mataga equation: ${ }^{32-35}$

$$
\bar{v}=-\frac{2\left(\Delta \mu_{\mathrm{e}}\right)^{2}}{h c s^{3}}\left[\frac{\epsilon_{\mathrm{s}}-1}{2 \epsilon_{\mathrm{s}}+1}-\frac{n^{2}-1}{4 n^{2}+2}\right]
$$

where $h, c$, and $s$ are Planck's constant, the speed of light, and the semimajor axis of an ellipsoidal cavity containing the emitter, respectively.

There are several reasons, however, why it is difficult to determine $\Delta \mu_{\mathrm{e}}$ for the emissive interchain species in conjugated polymer films. First and most important, any liquid applied to the film interacts only with chromophores near the surface, but the fluorescence from the film is dominated by the much larger fraction of chromophores residing in the film interior. To selectively capture the solvatochromically shifted emission from near the surface of the film, an imaging system with a very shallow depth of field is required. Near-field scanning optical microscopy (NSOM) provides exactly such a system because it samples the evanescent field in the near-zone, which decreases exponentially with distance from the probe. ${ }^{36-39}$ Second, the range of liquids available to vary the polarity around the chromophores is limited by the solubility of the sample: the measurements do little good if the solvent dissolves the polymer film and breaks up the interchain species. Fortunately, conjugated polymers such as MEH-PPV are highly insoluble in polar liquids such as ethylene glycol and acetonitrile, providing at least some range of polarity for solvatochromic investigation. Finally, there is a potential complication that depends on the ease of diffusion of the liquid into the polymer film, often referred to as "swelling" of the polymer. ${ }^{40-42}$ If the solvent molecules intercalate into the film, then not only could the interactions between chains be altered but also the emission collected in the near-field regime could contain components from liquid/bulk interactions, as well as the desired liquid/surface interaction. This possibility can be investigated by using the topographic information provided by NSOM to verify that no physical changes take place at the surface of the conjugated polymer film upon exposure to the solvent.

In this paper, we demonstrate that each of these potential difficulties can be overcome to successfully perform NSOM solvatochromism experiments and characterize the interchain species in conjugated polymer films. Extraction of $\Delta \mu_{\mathrm{e}}$ from interfacial solvatochromic emission data collected via NSOM, however, is complicated by the fact that the theory underlying eq 1 assumes that the emitting dipole is solvated uniformly by the environment. This assumption clearly fails for interfacial solvatochromism, because the emission is collected from chromophores that are solvated on one side by a high-polarity liquid and on the other side by a solid host with vastly different dielectric properties. This means that eq 1 is likely to underestimate $\Delta \mu_{\mathrm{e}}$ because only half of the medium surrounding the dipole has the dielectric characteristics of the solvent. Thus, a new analysis method is required to extract accurate $\Delta \mu_{\mathrm{e}}$ values for NSOM-collected interfacial solvatochromic spectral shifts.

Recently, Benjamin has developed a theory for the solvatochromism of chromophores at the boundary of two different dielectric media ${ }^{43}$ that is directly applicable to the problem of determining $\Delta \mu_{\mathrm{e}}$ for the emissive interchain species in conjugated polymer films. In Benjamin's theory, the interface is modeled as a mathematically sharp plane separating two different dielectric media. Two equal-sized spherical cavities 


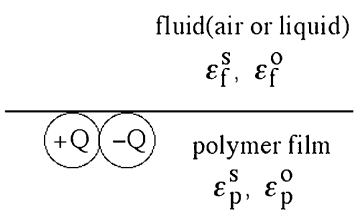

Figure 1. A schematic representation of the model (eq 2) used to calculate the solvatochromic shift of a chromophore at the film/liquid interface.

(radius $a$ ) separated by a distance $R$ are located just on the film side of the interface, as shown in Figure 1, and the solute dipole is represented as equal and opposite charges located at the center of each cavity. The solvatochromic shift associated with a change in the magnitude of this charge separation as a result of an electronic excitation can be found analytically in this model and is given by ${ }^{43}$

$$
\begin{gathered}
\Delta E_{\mathrm{g} \rightarrow \mathrm{e}}=h c \bar{v}=\left(Q_{\mathrm{e}}-Q_{\mathrm{g}}\right)^{2}\left(I_{\mathrm{o}}-I_{\mathrm{s}}\right)+\left(Q_{\mathrm{e}}-Q_{\mathrm{g}}\right)^{2}\left(I_{\mathrm{s}}-\frac{1}{a}\right) \\
I_{m}=\frac{1}{2 \epsilon_{\mathrm{p}}^{m}}\left[\frac{2}{a}-\frac{1}{R}+\left(\frac{\epsilon_{\mathrm{p}}^{m}-\epsilon_{\mathrm{f}}^{m}}{\epsilon_{\mathrm{p}}^{m}+\epsilon_{\mathrm{f}}^{m}}\right)\left(\frac{1}{a}-\frac{1}{\left(a^{2}+R^{2} / 4\right)^{1 / 2}}\right)\right]
\end{gathered}
$$

In eq 2, $\epsilon_{\mathrm{p}}^{m}$ and $\epsilon_{\mathrm{f}}^{m}$ are the dielectric constants of the polymer and the applied fluid (either air or liquid), respectively; the index $m=\mathrm{o}$, s stands for the optical and static values of the dielectric constant $\left(\epsilon^{\mathrm{o}}=n^{2}\right)$; and $Q_{\mathrm{g}}$ and $Q_{\mathrm{e}}$ are the magnitudes of the separated charges in the ground and excited states, respectively.

In the next section, we show how our NSOM solvatochromic method, in combination with eq 2 , allows us to accurately determine $\Delta \mu_{\mathrm{e}}$ for chromophores at the interface of polymer films. After describing our apparatus for performing NSOM solvatochromism experiments, we present measurements of the solvatochromically shifted interfacial emission spectra from a laser dye embedded in PMMA. Upon analyzing the data with eq 2 , we are able to successfully reproduce the literature value for the change in dipole moment of the dye, verifying the accuracy of the method. With our demonstrated ability to make accurate interfacial solvatochromic measurements, we then return to the main focus of this work, the solvatochromic study of the emissive interchain species in annealed films of poly(2methoxy-5-(2'-ethylhexyloxy)-1,4-phenylene vinylene) (MEHPPV). We chose to study annealed MEH-PPV films because they contain a much higher degree of interchain interactions than as-cast films. ${ }^{9,44}$

We find that even though the interfacial PL from annealed MEH-PPV films is spatially homogeneous in the absence of applied solvent, ${ }^{44}$ the emission becomes spatially inhomogeneous in the presence of polar solvents. The data show that the majority of the detected interfacial emission from the annealed film shifts to the blue with increasing solvent polarity, indicating that most of the interchain species in the annealed film are excimer-like in nature, with an excited state that is less-polar than the ground state. In a few spatially localized regions of the film, however, the emission shows a dramatic red shift with increasing polarity of the applied solvent. This is the hallmark of an increase in dipole moment upon excitation, consistent with a charge-separated interchain excited-state such as an exciplex or polaron pair. Upon analyzing the observed spectral shifts using eq 2 , we find a $\Delta \mu_{\mathrm{e}}$ of -4 to $-7 \mathrm{D}$ for the blue-shifted emission from the majority of the film, while the few regions that show red-shifted emission are consistent with a $\Delta \mu_{\mathrm{e}}$ of +9 to +13 D. Overall, the presence of solvatochromic emission shifts with different directions in different spatial regions clearly

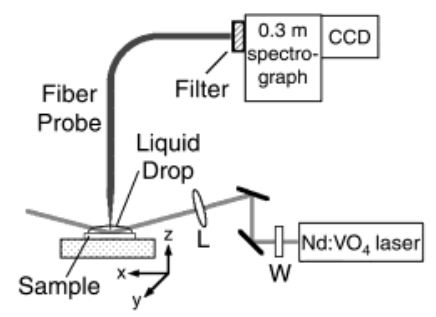

Figure 2. Experimental setup for performing PL NSOM solvatochromism measurements: (W) half-wave plate; (L) 8-cm focal length plano-convex lens. The 0.3-m spectrograph, 532-nm notch filter, and CCD used to acquire spectra were replaced with a red-extended PMT (Hamamatsu R3896) and appropriate filters for SRPL imaging. The excitation laser was incident on the sample at $\sim 65^{\circ}$ from normal.

shows that there are several types of emissive interchain species in films of conjugated polymers. The data strongly suggest that there is indeed a continuum of possible interchain excited states with differing extents of charge delocalization that depend sensitively on the local chain packing. Moreover, the fact that high degrees of charge separation are found only in spatially localized regions indicates that charge-separated interchain species may be associated with intrinsic defects. Finally, we find that the size of the solvatochromically red-shifted domains increases in films of low molecular weight MEH-PPV, implying that the spatial variation observed in dipole moments is intrinsic and possibly related to the presence of chain ends.

\section{Experimental Section}

MEH-PPV film samples ( $200 \mathrm{~nm}$ thick) were prepared by spin-casting $1 \%(\mathrm{w} / \mathrm{v})$ solutions of MEH-PPV in either chlorobenzene $(\mathrm{CB})$ or tetrahydrofuran $(\mathrm{THF})$ onto acid-cleaned glass substrates at room temperature under a nitrogen atmosphere. Under an inert atmosphere, the films were first heated to $50{ }^{\circ} \mathrm{C}$ for several hours to remove any remaining solvent and then annealed at $\sim 210{ }^{\circ} \mathrm{C}$ (above the glass transition temperature $^{9}$ ) for more than $8 \mathrm{~h}$. No differences were observed between annealed films cast from either CB or THF, consistent with the idea that the annealing process removes all memory of the initial as-cast chain packing. ${ }^{9,44}$ The molecular weight of the polymer used in the bulk of the experiments was determined by gel permeation chromatography (using polystyrene as a reference) to be $450000 \mathrm{Da}$. For a few experiments where noted, a low molecular weight batch of $60000-\mathrm{Da} \mathrm{MEH}-\mathrm{PPV}$ was used.

The NSOM system (Thermomicroscopes, Lumina), which is equipped with a noncontact ( $\sim 5-10 \mathrm{~nm}$ separation) tuningfork-based shear-force feedback mechanism that we employ for near-field spatially resolved photoluminescence (SRPL) measurements of annealed MEH-PPV films, has been described previously. ${ }^{38,45}$ Chemically etched $\mathrm{SiO}_{2}$ nonmetal-coated fiber optic probes were produced with a $\sim 75 \mathrm{~nm}$ diameter tip and were mounted so as to overhang the end of the tuning fork by about $0.6 \mathrm{~mm}$ as described by Lee et al. ${ }^{46}$ Stable near-field feedback is facilitated with this overhanging probe design because in the solvatochromic measurements the tuning fork does not contact the liquid: only the fiber optic probe is immersed, which helps to maintain the high Q-factor that is essential for noncontact scanning. To prevent photooxidation of the sample, the entire NSOM system was sealed in a dry nitrogen purge box.

Figure 2 shows a schematic of the experimental design. Nearfield measurements were conducted in "collection mode" geometry with the optical excitation incident upon the sample $60^{\circ}$ from normal with vertical polarization at the position where 
the fiber probe collected the emission. To maintain a constant tip/field geometry, the NSOM probe remained static during all measurements; spatial motion was performed by scanning the sample stage in the $x$ and $y$, as well as the $z$ (feedback), directions. A diode-pumped, frequency-doubled $\mathrm{Nd}: \mathrm{VO}_{4}$ laser (Spectra-Physics) $(40 \mu \mathrm{W}, 532 \mathrm{~nm}, \mathrm{CW})$ was used as the excitation source. The laser beam was focused to an $\sim 400-\mu \mathrm{m}$ diameter spot on the sample at the position of the NSOM probe. Optical signals were collected by the near-field probe and directed via the fiber optic either to long pass filters and a redextended photomultiplier (Hamamatsu R3896) for SRPL imaging or to a 532-nm holographic notch filter and a $0.3-\mathrm{m}$ spectrograph to generate SRPL spectra. In the spectrograph, the collected emission was dispersed with a $150-\mathrm{gr} / \mathrm{mm}$ grating and was integrated for $15 \mathrm{~s}$ for MEH-PPV (25 s for DCM/PMMA) with a nitrogen-cooled, back-illuminated CCD camera (Roper Scientific). All of the images presented below are $200 \times 200$ pixel arrays.

A. The NSOM Interfacial Solvatochromism Method. The procedure for obtaining SRPL solvatochromism information was straightforward. First, a few drops of the selected organic liquid were placed on top of the film sample; then the NSOM probe was brought into near-field feedback. Second, the excitation laser power was adjusted to compensate for reflection losses at the interface between the different liquids and air so as to maintain constant excitation intensity on the sample at the buried liquid/ solid interface. Third, the laser alignment was adjusted to compensate for parallax due to the liquid, and finally the solvatochromically shifted emission spectra were collected. A large number of SRPL spectra were collected from many regions of multiple film samples; the results for each type of sample were similar to the representative spectra shown in the figures below. The high volatility of most of the liquids that we used made stable near-field feedback over periods of $>10 \mathrm{~min}$ (which is required for imaging) difficult. A notable exception was ethylene glycol (EG), which has such a low vapor pressure at room temperature that stable feedback was possible for many hours, despite the presence of the nitrogen purge. This is the reason that we chose to present images of film samples under EG or nitrogen gas in the results shown below.

To confirm our assignment of the observed spectral shifts to solvatochromism, we measured SRPL spectra and film topographies under nitrogen gas for each region both before and after the application of each liquid. The acquired SRPL spectra and topographies were completely reproducible as a function of time, confirming the lack of photodamage and the absence of polymer swelling during the course of the experiments. As shown, respectively, in Figure $3 a-c$ for annealed MEH-PPV films, topographical data acquired before application of EG, while EG was in contact with the film, and after removal of the highpolarity organic solvent do not reveal any detectable morphology changes within the $z$-precision of our noncontact NSOM $(\sim 0.6$ $\mathrm{nm})$. The increased noise levels in Figure $3 \mathrm{~b}$, in which the liquid is present on the film, reflect the somewhat decreased sensitivity of our noncontact feedback mechanism in the presence of the liquid. Despite the increased feedback noise, new topographical features are not observable in the image. For MEH-PPV film samples, organic liquids with dielectric constants lower than that of propionitrile (e.g., acetone) caused the films to noticeably swell, as shown in Figure $3 \mathrm{~d}$. Thus, we did not employ any such liquids in the experiments on MEH-PPV films described below.

To verify that the observed spectral shifts in the SRPL measurements are due to changes in the electronic environment a) topog before EG

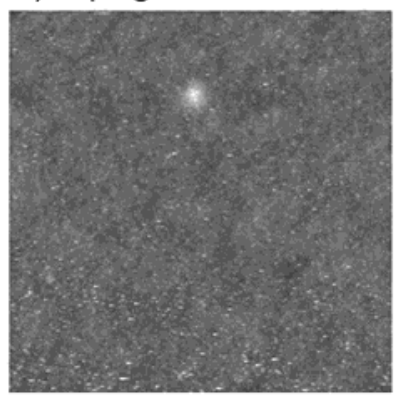

c) topog after EG

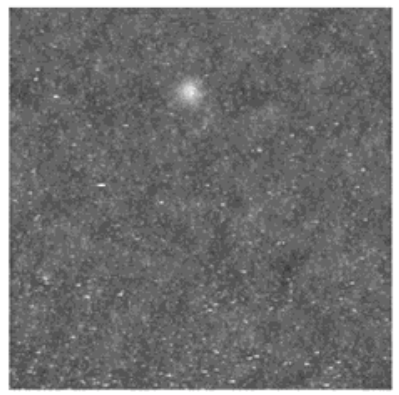

b) topography w/EG

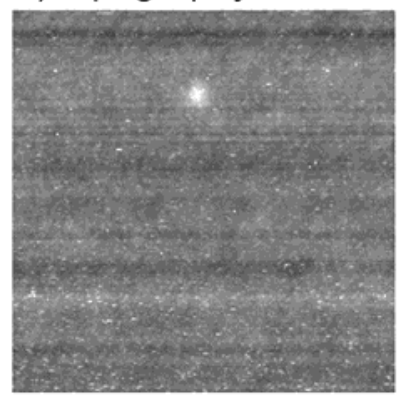

d) topog after acetone

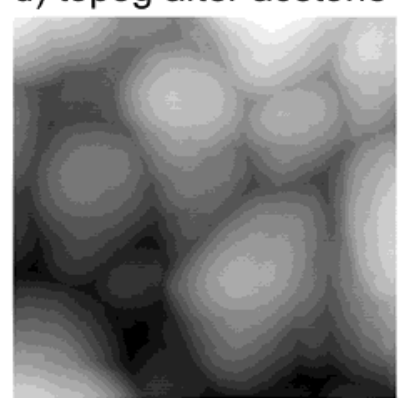

Figure 3. Topographic investigation of swelling of annealed MEHPPV films by high-polarity liquids. Each image shows the same (10 $\mu \mathrm{m})^{2}$ area of a film collected (a) before application of ethylene glycol (EG), (b) with EG, and (c) after removal of EG from the film surface. Panels $\mathrm{a}-\mathrm{c}$ are shown using the same $z$ scale with a maximum height of $19 \mathrm{~nm}$. High-polarity liquids such as EG cause no detectable change in topography. Lower-polarity liquids, such as acetone, caused the sample to swell quite noticeably (panel d) (image collected on a different $(10 \mu \mathrm{m})^{2}$ area of the film). The $z$ scale of panel d represents a height change of $\sim 175 \mathrm{~nm}$.

surrounding the polymer chromophores near the film's surface and not due to physical changes in bulk polymer chain packing, we also performed far-field confocal PL microscopy experiments. These experiments were identical to those conducted in the near-field, with emission collected from the same annealed MEH-PPV films; data in the absence and presence of organic liquids are shown in Figure $4 a, b$, respectively. For these farfield experiments, the films are physically thinner than the $z$-resolution $(\sim 500 \mathrm{~nm})$ of the confocal microscope. Thus, the imaged PL is collected from the entire thickness of the film, resulting in a noninterfacial bulk measurement. When the highpolarity liquids were applied on the polymer films, no significant changes in PL were observed: the variations in the optical signals as a function of spatial position were $<1 \%$ in amplitude. This verifies that the spectral changes that we observed in the near-field do not constitute a bulk phenomenon. Application of lower polarity liquids (e.g., acetone) that altered the film morphology by swelling resulted in confocal images that have noticeable contrast $(\geq 20 \%)$, as shown in Figure 4c.

It is worth noting that, if the observed red-shifting solvatochromic domains in annealed MEH-PPV films discussed below were the result of spatially varying swelling, the presence of spatially varying swelling would still constitute a previously undiscovered form of inhomogeneity in the chain packing of annealed conjugated polymer films. We believe that such a difference in chain packing, however, would have been detectable in the emission spectra even in the absence of applied liquid. Both our previously reported SRPL studies in the absence of solvent ${ }^{44}$ and the data presented below show essentially identical SRPL spectra at all measured positions in the annealed 
a) $\mathrm{PL} w / \mathrm{N}_{2}$

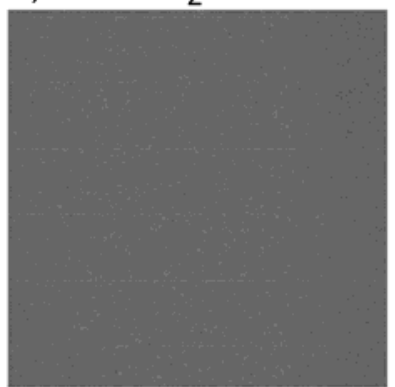

b) PL w/EG

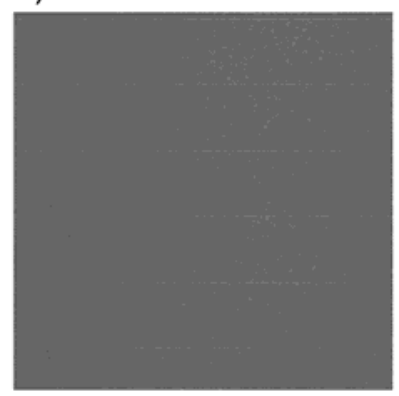

c) PL w/acetone

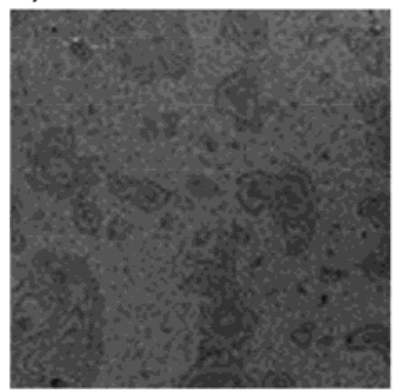

Figure 4. Far-field confocal photoluminescence measurements on annealed MEH-PPV films in contact with (a) air, (b) EG, and (c) acetone. Excitation was provided by the 543-nm line of a $\mathrm{He}-\mathrm{Ne}$ laser, and emission was collected at wavelengths longer than $700 \mathrm{~nm}$. Because the $\sim 200$-nm thick films are thinner than the $z$ resolution of the confocal microscope $(\sim 500 \mathrm{~nm})$, the emission is collected from the entire thickness of the film and therefore constitutes a bulk measurement. The variation of the optical signals is $<1 \%$ in panels a and $\mathrm{b}$. A much larger optical signal level variation $(\geq 20 \%)$ is observed in panel c, under the same conditions for which the topographical data in Figure $3 \mathrm{~d}$ show the film to be swollen with the solvent. The field of view in each panel is $(30 \mu \mathrm{m})^{2}$. These far-field measurements demonstrate that the domains observed in the NSOM studies of MEH-PPV films, such as those in Figure 7d, are observable due to the very shallow depth of NSOM.

films, verifying that the changes measured upon the application of solvent are due to solvatochromism.

B. Demonstration of the Interfacial Solvatochromism Method: DCM in PMMA. To assess whether our NSOM interfacial solvatochromism measurements, in combination with eq 2 , are capable of accurately determining $\Delta \mu_{\mathrm{e}}$, we have performed a series of calibration experiments on films of a laser dye embedded in an inert polymer host. The chromophore that we have chosen, trans-4-dicyanomethylene-2-methyl-6- $p$-dimethylaminostyryl-4H-pyran (DCM) (see Figure 5 for chemical structure), has been well studied in noninterfacial solvatochromic measurements and has known dipole moments in the ground and charge-separated excited state of 6.1 and $26 \mathrm{D}$, respectively. ${ }^{47,48}$ Our calibration samples consisted of 1:100 by weight films of DCM dispersed in 996000-Da average molecular weight poly(methyl methacrylate) (PMMA). The samples were produced by stirring a solution of DCM and PMMA in dichloromethane for $30 \mathrm{~min}$ before drop-casting the mixture onto acid-cleaned substrates. Interfacial emission spectra acquired (as described above) from the DCM/PMMA films placed in contact with both air and water are shown in Figure 5. The SRPL spectra collected with the dye sample in contact with air and water exhibit emission maxima at 587.1 and $597.6 \mathrm{~nm}$, respectively. The integrated intensity of the emission spectra collected under both air and water was identical to within $\sim 1 \%$; this lack of intensity change upon application of polar liquid is

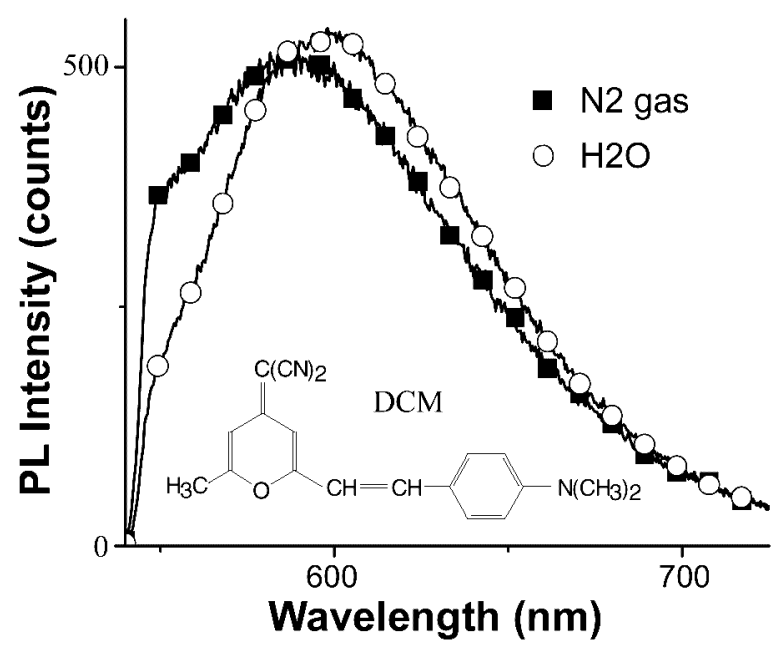

Figure 5. Solvatochromic behavior of the SRPL spectra of the laser dye DCM in PMMA for the film in contact with (ם) no solvent $/ \mathrm{N}_{2}$ gas and $(O)$ water. The DCM emission spectrum clearly shifts to lower energy when the film is placed in contact with water. Input of the measured peak positions from the two spectra into eq 2 closely reproduces the literature value for the change in dipole moment between the ground and excited states of DCM (see text). The inset shows the chemical structure of DCM.

TABLE 1: Static and Optical Dielectric Constants at 293 $K^{29,72}$ for the Materials Used in This Study ${ }^{a}$

\begin{tabular}{lcc}
\hline \multicolumn{1}{c}{ material } & $\epsilon^{\mathrm{S}}$ & $\epsilon^{\mathrm{O}}$ \\
\hline water & 78 & 1.777 \\
ethylene glycol (EG) & 37.7 & 2.051 \\
propionitrile (PrCN) & 28.9 & 1.866 \\
1:1 PrCN/MeCN & 32.4 & 1.836 \\
methanol & 32.7 & 1.764 \\
acetonitrile (MeCN) & 35.9 & 1.806 \\
PMMA & 3.6 & 2.199 \\
nitrogen gas & 1.0 & 1.000
\end{tabular}

${ }^{a}$ Optical dielectric constants were taken from the literature for a wavelength of $589 \mathrm{~nm}$.

also characteristic of the experiments performed on annealed MEH-PPV films, described below.

The shift to lower energy of the near-field detected interfacial emission spectra collected from DCM/PMMA films when the external medium is changed from air to water fits perfectly with the reasoning presented in the Introduction: water is more effective at stabilizing the large excited-state dipole of DCM than the smaller ground-state dipole, leading to red-shifted emission. Using the measured peak positions of the interfacial emission $(\bar{v})$ and the known static and dielectric constants for air, water, and PMMA (listed in Table 1) in eq 2, we were able to reproduce the literature values for the $\Delta \mu_{\mathrm{e}}$ of DCM for the choice of cavity radius (a) (cf. Figure 1) of $4.15 \AA$ and $R=$ $2 a{ }^{49}$

We note that our best fit value of $4.15 \AA$ for the radius of the spherical cavities $(a)$ in eq 2 is almost exactly half the published value of the size of the emitter cavity $(s)$ used for this dye in conjunction with eq $1(8 \AA))^{47,48}$ This factor of 2 difference in cavity size between eq 1 and eq 2 makes sense in light of how each model is constructed: the dual sphere model of eq 2 has the same effective spatial charge separation as the single-sphere model of eq 1 when the radius of the single sphere is twice that of the double sphere. The fact that the calculated spatial extent of charge separation is so similar between the two models is reassuring, because the primary source of error in the analysis of any solvatochromic data results from 
uncertainty in choosing the size of the emitting cavity in the model. ${ }^{50}$ This is because there is no a priori method for assigning the radii of the spheres $(a)$ used in eq 2 (or the size of the emitter $(s)$ in eq 1) based on the molecular geometry, even for simple molecular chromophores. We expect that the choice of cavity radius should be even more uncertain in complicated systems such as conjugated polymer films, where the possibility of a distribution of emitter sizes cannot be ruled out because of the presence of both intra- and interchain emissive species. With these caveats concerning the cavity size in mind, we turn in the next section to the application of our interfacial solvatochromic method to the interchain emission from annealed $\mathrm{MEH}-$ PPV films.

\section{Solvatochromism of Interchain Emission from Annealed MEH-PPV Films}

In recent collaborative work, ${ }^{44}$ the UCLA and Berkeley groups studied the emission from films of MEH-PPV using NSOM. In agreement with the results of others, ${ }^{17,51-55}$ we demonstrated that the optical properties of conjugated polymer films are spatially inhomogeneous on nanometer length scales. We also found a correlation between topographic features on the surface of the films and the presence of more tightly packed polymer chains, as characterized by a red shift of the SRPL spectrum and a decreased photooxidation rate. When the MEHPPV films were thermally annealed (i.e., heated above the polymer glass transition temperature for several hours), however, the film topography became smooth on the nanometer scale and there was no evidence for inhomogeneity in either the luminescence properties or the photodamage rate. The flatter topography of the annealed films results from the increased mobility of the polymer strands above the glass transition temperature, allowing the polymer chains to flow into a smoother, more even morphology. The loss of inhomogeneity also results from the increased chain mobility in the polymer melt; the annealed chains favor packing into thermodynamically low-energy states, decreasing the number of chromophores with high-energy twisted or kinked structures. This packing of the chains into low-energy structures also greatly increases the concentration of interchain species, as evidenced by a large redshift of the luminescence spectrum and a decrease of the PL quantum yield. ${ }^{9,44}$

Our selection of annealed MEH-PPV films for this solvatochromism study provides two major advantages. First, the majority of the emission from annealed films is interchain, facilitating solvatochromic determination of the change in dipole associated with production of the interchain species. Second, the topography of annealed MEH-PPV films is essentially featureless, providing a convenient reference for the detection of swelling or other unwanted variations in the local chain packing.

A. Spatially-Varying Solvatochromism in Annealed MEHPPV Films. Figure 6a,b presents representative SRPL spectra from an annealed MEH-PPV film that were collected at two different fixed positions a few micrometers apart in various solvent environments. The filled squares/heavy solid curves display the spectra taken under nitrogen with no solvent present. Consistent with our previous report, ${ }^{44}$ the spectra from the two different regions are identical, indicating that the PL of the annealed film is spatially homogeneous. The open circles/light gray curves show the SRPL spectrum from the same two regions with the film under ethylene glycol, while the solid triangles/ light solid curves show the SRPL from the two regions under acetonitrile $(\mathrm{MeCN})$. For the region studied in Figure 6a, the
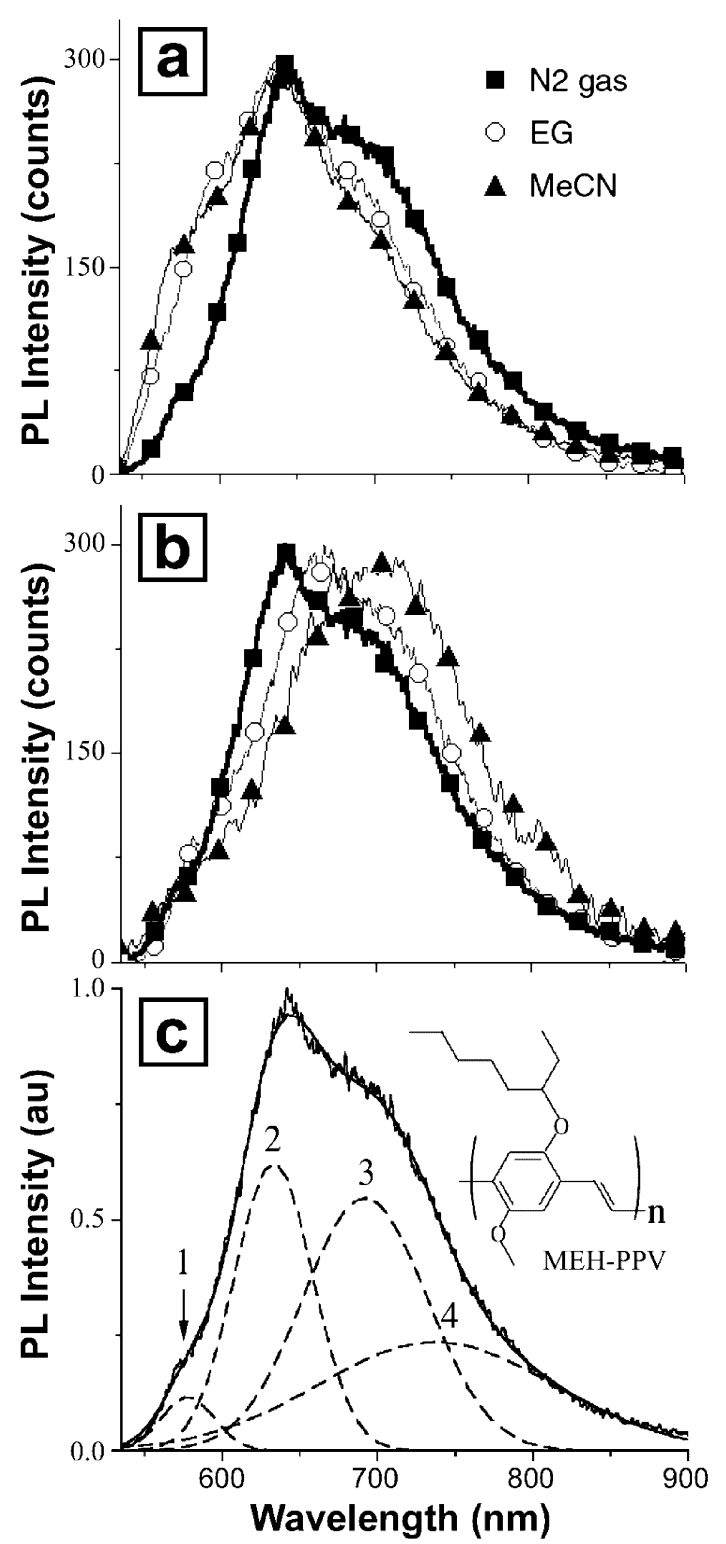

Figure 6. Solvatochromic behavior of the SRPL spectra of annealed MEH-PPV films under different solvents: (ם) no solvent/ $\mathrm{N}_{2}$ gas; $(\mathrm{O})$ ethylene glycol; ( $\mathbf{\Lambda})$ acetonitrile. Part a shows a region for which the SRPL blue shifts with polar liquids, typical of $>95 \%$ of monitored regions; part b shows the SRPL for one of the $<5 \%$ of the regions for which the SRPL red shifts with increasing liquid polarity. Spectra collected from different spatial positions with no solvent on the film surface were indistinguishable. Part c shows a representative fit of an SRPL spectrum to the sum of four Gaussian bands (three bands could not accurately reproduce the observed spectra). The chemical structure of MEH-PPV is shown in the inset.

emission spectrum is observed to shift slightly to the blue (relative to that under nitrogen gas) upon the application of polar liquids. Figure $6 \mathrm{~b}$ clearly displays the opposite trend, with the SRPL spectrum from this region shifting significantly to the red when the high-polarity liquids were applied. This spatially varying solvatochromic behavior is completely reversible upon removal of the applied solvent and indicates that a previously undiscovered form of inhomogeneity persists in films of MEHPPV, even after the annealing process.

After sampling many regions of multiple films, we found that the blue solvatochromic shift observed in Figure 6a is representative of the majority of the emission: $>95 \%$ of the sampled regions show this blue-shifting solvatochromic behavior. The 


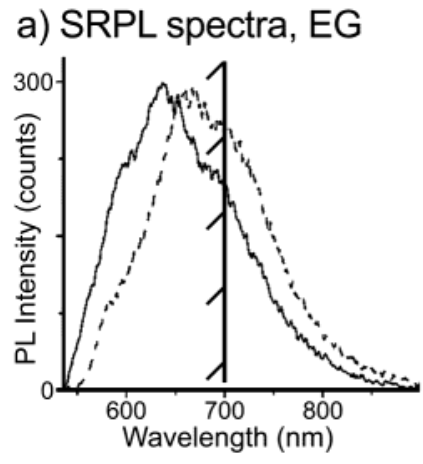

b) Topograpghy
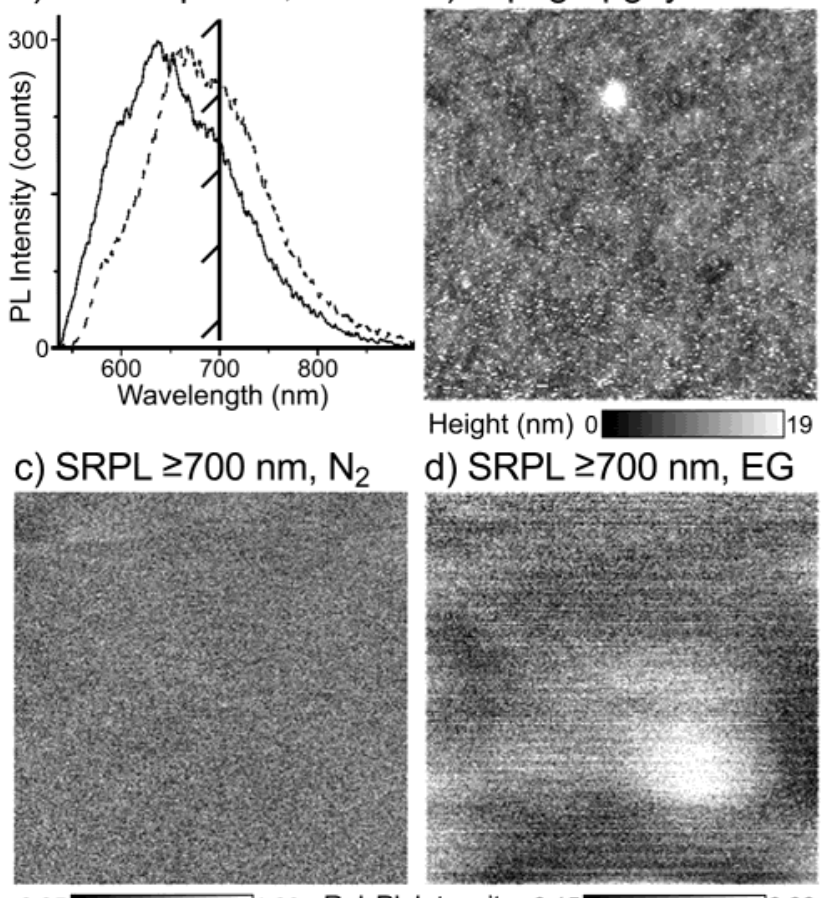

0.95

1.00 Rel. PL Intensity 0.15

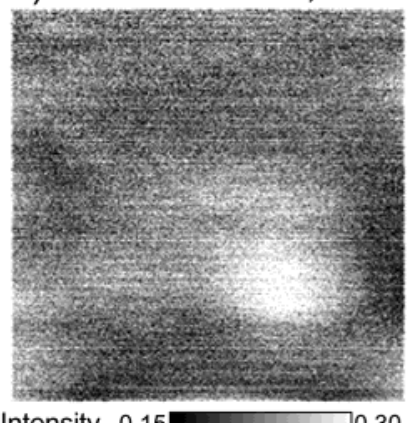

Figure 7. NSOM images of the solvatochromically red-shifted domains in annealed MEH-PPV films. Part a compares the SRPL under ethlyene glycol (EG) from different spatial regions; the solid curve is the same as that in Figure 6a; the dashed curve is the same as that in Figure 6b. The integrated PL intensities (integrated from 535 to $900 \mathrm{~nm}$ ) for all regions under all of the applied liquids were identical to $\pm 1 \%$, but there is $\sim 40 \%$ less total integrated intensity at wavelengths $\geq 700 \mathrm{~nm}$ from regions that show the solvatochromic red shift. Part $b$ shows the topography of a typical $(10 \mu \mathrm{m})^{2}$ region. Parts c (no solvent) and d (EG) show scanning SRPL images over the same region as part $b$ when only the $\geq 700$-nm emission was collected. The contrast in part $\mathrm{d}$ delineates the spatial extent of one of the larger red-shifting solvatochromic regions. No contrast was observed for images in which the entire PL was collected (not shown; cf. ref 44).

solvatochromic red shift observed in Figure $6 \mathrm{~b}$ is much less typical and is observed from $<5 \%$ of the film's area. Figure $7 \mathrm{a}$ replots the spectra shown in Figure $6 a, b$ for the two different regions of the annealed MEH-PPV film under EG on an absolute PL intensity scale. This direct comparison shows that one manifestation of the spatial solvatochromic inhomogeneity is a large difference in integrated emission intensity to the red of $\sim 700 \mathrm{~nm}$. In other words, there is a $\sim 40 \%$ reduction in integrated intensity from the blue-shifting region studied in Figure 6 a relative to the red-shifting region shown in Figure $6 \mathrm{~b}$, despite the fact that the total integrated PL from each region is the same to within $1 \%$. This makes it possible to spatially image the regions of the film that exhibit the solvatochromic red shift by scanning the NSOM tip and using an optical filter to detect only wavelengths longer than $700 \mathrm{~nm}$.

The results of this type of imaging experiment are shown in Figure $7 b-d$, which displays the topography (Figure $7 b$ ), the $\geq 700 \mathrm{~nm}$ SRPL under nitrogen (Figure 7c), and the $\geq 700 \mathrm{~nm}$ SRPL under EG (Figure $7 \mathrm{~d}$ ), all for the same $(10 \mu \mathrm{m})^{2}$ region. The topography is nearly featureless and very flat, and the $\geq 700$ nm SRPL scanned under nitrogen gas shows no perceptible contrast, in agreement with our previous SRPL results on annealed MEH-PPV films. ${ }^{44}$ When EG is present on the film's surface, however, the $\geq 700 \mathrm{~nm}$ SRPL shows strong contrast, revealing roughly circular spatially localized regions that are
TABLE 2: Average Dipole Moment Change upon Excitation Calculated from the Interfacial Continuum Model (Eq 2) for Both Solvatochromically Blue-Shifting (e.g., Figure 6a) and Solvatochromically Red-Shifting Regions (e.g., Figure 6b) of Annealed MEH-PPV Films

\begin{tabular}{cccccc}
\hline & \multicolumn{2}{c}{$\begin{array}{c}\text { avg } \Delta \mu_{\mathrm{e}}(\mathrm{D}) \text { for } \\
\text { blue-shifting regions }\end{array}$} & & \multicolumn{2}{c}{$\begin{array}{c}\text { avg } \Delta \mu_{\mathrm{e}}(\mathrm{D}) \text { for } \\
\text { red-shifting regions }\end{array}$} \\
\cline { 2 - 3 } \cline { 5 - 6 }$(\mathrm{nm})$ & $\epsilon_{\mathrm{p}}^{\mathrm{s}}=4$ & $\epsilon_{\mathrm{p}}^{\mathrm{s}}=5$ & & $\epsilon_{\mathrm{p}}^{\mathrm{s}}=4$ & $\epsilon_{\mathrm{p}}^{\mathrm{s}}=5$ \\
\hline 577 & 2.0 & 2.2 & & -2.4 & -2.8 \\
633 & -6.4 & -7.3 & & 8.7 & 10.0 \\
692 & -7.6 & -8.7 & & 11.5 & 13.2 \\
738 & -4.3 & -5.0 & & 13.2 & 15.2
\end{tabular}

$1-2 \mu \mathrm{m}$ in diameter. The bright region in Figure $7 \mathrm{~d}$ shows one of the larger domains that we observed with a $\sim 2 \mu$ m diameter; a more representative sample of regions that exhibit the red solvatochromic shift is shown below in Figure 9a. The majority of the areas investigated, however, show no such contrast and display only the slight blue-shifting solvatochromism. We emphasize that the features observed in these images do not result from effects such as differential quenching of emission by the applied liquid, because the overall integrated PL intensity (from 535 to $900 \mathrm{~nm}$ ) is identical within $\pm 1 \%$ for the entire scanned region.

The blue shift with increasing liquid polarity seen in Figure $6 \mathrm{a}$ indicates that the electronic ground state of most of the chromophores in annealed MEH-PPV films possesses a larger dipole moment than their excited states. For the excited state to have a smaller dipole moment than the ground state, the excited state must have very little charge separation, typical of what is expected for an excimer or weakly charge-separated exciplex. ${ }^{56}$ The few regions such as those in Figure $6 \mathrm{~b}$ (and highlighted in Figure 7d) that exhibit red shifts are indicative of excited states with increased charge separation relative to the ground state. This is what is expected for interchain species such as highly asymmetrical exciplexes or polaron pairs.

B. Continuum Electrostatic Modeling of MEH-PPV Interfacial Solvatochromism. We can use data such as that shown in Figure 6, along with eq 2, to estimate quantitatively the change in dipole moment upon excitation in the different spatial regions of annealed MEH-PPV films. To characterize the spectral data and determine $\bar{v}$, we fit the observed SRPL spectra to the sum of four Gaussian bands, as shown in Figure $6 \mathrm{c}$, which with no applied solvent have peaks at 577, 633, 692, and 738 $\mathrm{nm}$ (and fwhm of 14.3, 25.5, 40.2, and $70.1 \mathrm{~nm}$, respectively). Our choice to fit the spectra with independent Gaussians was made in part to allow for the possibility that the observed emission from the annealed films could contain both intra- and interchain components that might have independent solvatochromic shifts. Other than allowing for this possibility, our fit to four independent Gaussians is not intended to imply any specific physical model for the emission. ${ }^{9}$

To determine the difference in charge separation between the ground and excited states, we employ a value of $\epsilon_{\mathrm{p}}^{\mathrm{o}}=3.61$ for the optical dielectric constant of MEH-PPV, as determined from measurements of the index of refraction of MEH-PPV at 632 nm. ${ }^{57,58}$ Because the static dielectric constant of the polymer is unknown but must be greater than the value of the optical dielectric constant, we have performed the analysis using values of both 4 and 5 for $\epsilon_{\mathrm{p}}^{\mathrm{s}}$. The static and optical dielectric constants of the applied liquids needed to solve eq 2 for $\Delta \mu_{\mathrm{e}}$ are presented in Table 1 . Using all of these parameters, we solved eq 2 numerically, and the results are collected in Table 2 , which shows the change in dipole for our two choices of the polymer's static dielectric constant and an assumed value of $R$ 
TABLE 3: Calculated Changes in Dipole Moment for Blueand Red-Shifting Regions of Annealed MEH-PPV Films for Two Different Cavity Separations with $R=\mathbf{2 a}$

\begin{tabular}{|c|c|c|c|c|}
\hline \multirow{2}{*}{$\begin{array}{l}\text { band center } \\
(\mathrm{nm})\end{array}$} & \multicolumn{2}{|c|}{$\begin{array}{c}\text { avg } \Delta \mu_{\mathrm{e}}(\mathrm{D}) \text { for } \\
\text { blue-shifting regions, } \\
\epsilon_{\mathrm{p}}^{\mathrm{s}}=4\end{array}$} & \multicolumn{2}{|c|}{$\begin{array}{c}\text { avg } \Delta \mu_{\mathrm{e}}(\mathrm{D}) \text { for } \\
\text { red-shifting regions, } \\
\epsilon_{\mathrm{p}}^{\mathrm{s}}=4\end{array}$} \\
\hline & $R=5$ & $R=8$ & $R=5$ & $R=8$ \\
\hline 577 & 2.0 & 4.1 & -2.4 & -4.9 \\
\hline 633 & -6.4 & -12.9 & 8.7 & 17.6 \\
\hline 692 & -7.6 & -15.4 & 11.5 & 23.3 \\
\hline 738 & -4.3 & -8.75 & 13.2 & 26.6 \\
\hline
\end{tabular}

TABLE 4: Measured Shifts of the Near-Field Detected PL for Annealed MEH-PPV Films under Nitrogen Relative to the Far-Field Detected PL (cf. Figure 8a) ${ }^{a}$

\begin{tabular}{ccccc}
\hline \multirow{2}{*}{$\begin{array}{c}\text { near-field } \\
\text { band center }\end{array}\left(\begin{array}{c}\text { far-field } \\
\text { band center }\end{array}\right.$} & $\begin{array}{c}\text { measured } \\
(\mathrm{nm})\end{array}$ & \begin{tabular}{c} 
shift $\left(\mathrm{cm}^{-1}\right)$ \\
\cline { 4 - 5 }
\end{tabular} & $\epsilon_{\mathrm{p}}^{\mathrm{s}}=4\left(\mathrm{~cm}^{-1}\right)$ & $\epsilon_{\mathrm{p}}^{\mathrm{s}}=5\left(\mathrm{~cm}^{-1}\right)$ \\
\hline 577 & 580 & -89.6 & -32 & -50.5 \\
633 & 624 & 228 & 325 & 556 \\
692 & 670 & 475 & 458 & 790 \\
738 & 724 & 262 & 147 & 261
\end{tabular}

${ }^{a}$ The band shifts of the far-field PL spectrum were also predicted from eq 1 (which is equivalent to eq 2 in the absence of the interface) using the calculated values of $\Delta \mu_{\mathrm{e}}$ generated from the near-field solvatochromic measurements (Table 2) and an MEH-PPV static dielectric constant $\epsilon_{\mathrm{p}}^{\mathrm{s}}$ of either 4 or 5 . The closest match between the measured and calculated band shifts (cf. Figure $8 b$ ) results from the choice of $\epsilon_{\mathrm{p}}^{\mathrm{s}}=4$ and $R=5 \AA$.

$=2 a=5 \AA$. Table 2 shows that the calculated magnitude of the change in dipole increases by $\sim 15 \%$ as $\epsilon_{\mathrm{p}}^{\mathrm{s}}$ is increased from 4 to 5 . The calculated values for $\Delta \mu_{\mathrm{e}}$ increase by a factor of 2 when the radius of the emitter is increased from $R=2 a=$ 5 to $R=2 a=8 \AA$, as can be seen in Table 3 .

As an internal consistency check of our use of the interfacial solvatochromism theory for annealed MEH-PPV films, we used the calculated changes in dipole moment generated with eq 2 (Table 2, blue-shifting region with $R=2 a=5 \AA$ and $\epsilon_{\mathrm{p}}^{\mathrm{s}}=4$ D) to predict the spectral emission shift, $\bar{v}$, for the special case that the medium on both sides of the interface was MEH-PPV: in other words, we "removed" the interface by assuming identical dielectric properties on both sides of the dividing plane. This procedure allows us to use either eq 1 or eq 2 to compute the spectral position of the PL for bulk annealed films given the change in dipole and the PL spectra measured at the different polymer/liquid interfaces. The results of this calculation are summarized in Table 4 and Figure 8, which compares the interfacial (near-field) and bulk (far-field) PL collected from the same annealed MEH-PPV film. The calculated solvatochromic spectral shift for the bulk PL is in excellent agreement with the observed far-field PL spectrum (Figure 8b); in other words, the same $\Delta \mu_{\mathrm{e}}$ can explain all of the measured interfacial solvatochromic shifts and the observed difference between the interfacial and bulk PL spectra. The agreement is remarkably good given that the calculation does not account for the small fraction of red-shifting regions present throughout the bulk of the film. We also note that the agreement between the two sets of observations is much better for the choice of $\epsilon_{\mathrm{p}}^{\mathrm{s}}=4$ (rather than $\epsilon_{\mathrm{p}}^{\mathrm{s}}=5$ ) and $R=5 \AA$ (rather than $R=8 \AA$ ), suggesting that these are the best values for accurately describing the dielectric properties of MEH-PPV. Thus, the fact that the same set of calculated dipole changes can explain the results of two independent experiments leads us to believe that eq 2 (with the $\epsilon_{\mathrm{p}}^{\mathrm{s}}=4$ parameters in Table 2) provides an internally consistent
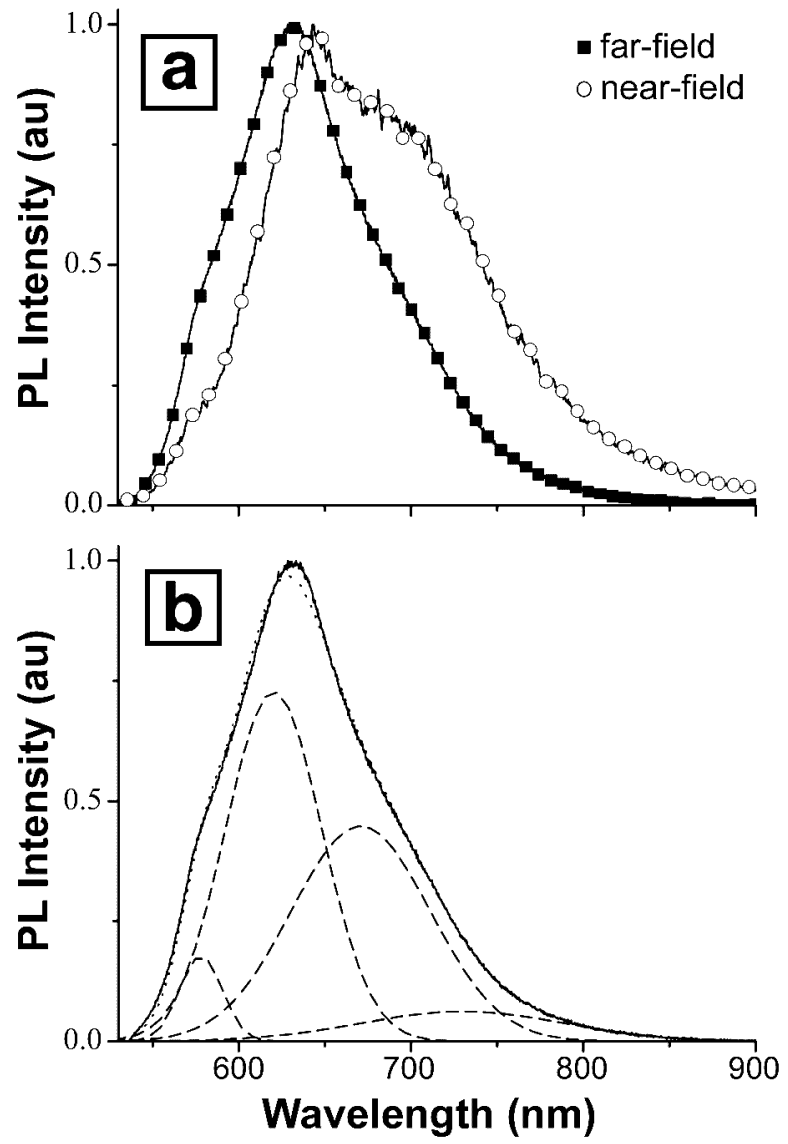

Figure 8. Prediction of the far-field PL spectrum from interfacial solvatochromic data. Part a shows the PL collected either (ם) in the far-field or $(O)$ in the near-field from annealed MEH-PPV films. The dipole moment changes generated with eq 2 and $\epsilon^{\mathrm{S}}=4$ (Table 2) were used to predict the spectral band positions of the far-field PL spectrum by assuming that the medium surrounding the dipole had the dielectric characteristics of the annealed MEH-PPV on both sides of the interface (Table 4). Part b shows that the sum (dotted curve) of four individual Gaussians (dashed curves) with band centers fixed at the predicted values closely reproduces the far-field PL spectrum (solid curve). In comparing the calculated to measured bulk PL spectrum, the widths of the four Gaussians were fixed at the same values as those presented in section III B, while the amplitudes of each band were allowed to vary as fitting parameters. Much of the dotted (calculated) trace is obscured by the solid (experimental) trace.

description for both the interfacial and bulk solvatochromism of annealed MEH-PPV.

Table 2 also shows that in both the red- and blue-shifting regions of the annealed film the solvatochromic shift of the three reddest Gaussian bands are nearly identical, while that of the bluest Gaussian band at $577 \mathrm{~nm}$ is different. The spectral position of the 577-nm peak suggests that it results from intrachain exciton emission rather than from an interchain species. The lack of significant solvatochromism for this band suggests either that there is little dipole change associated with the creation of an exciton or that the majority of the collected exciton emission comes from regions below the surface of the film that are not interacting with the solvent. Whatever the reason, the different sign and magnitude of the measured spectral shift and calculated dipole change for this bluest band support our view that it results primarily from intrachain exciton emission, while the three redder bands reflect the solvatochromism associated with the emission of interchain electronic species. Because each of the three interchain emission bands is observed to shift by a slightly different amount, we take the 
spread in the observed shifts as a rough measure of the uncertainty in our calculated value of $\Delta \mu_{\mathrm{e}}$.

In addition to the uncertainties inherent in measuring the individual band shifts, another difficulty in calculating the precise magnitude of the change in dipole upon excitation is that the static dielectric constants of the liquids used in the study are all of similar magnitude. This makes the determination of the change in dipole quite sensitive to the measured value of the spectral shift in air. Thus, it would be highly desirable to conduct the experiment with a liquid of significantly different dielectric constant to improve the accuracy of the fit. As mentioned above, however, solvents with lower dielectric constants than those chosen in Table 1 cause swelling of annealed MEH-PPV films, as shown in Figures 3d and 4c. Not only are we missing low-polarity solvents, but the high-polarity solvents that we were able to employ are, for the most part, capable of hydrogen bonding. Recent experiments have shown that site-specific interactions of the solvent with the interfacial chromophore can result in larger spectral shifts than are predicted by dielectric continuum theories, particularly when the solvent is capable of hydrogen bonding. ${ }^{59}$ Thus, the possibility of specific interfacial interactions altering the calculated values of $\Delta \mu_{\mathrm{e}}$ warrants further investigation. Indeed, parameters for performing MD simulations of conjugated polymers including a quantum mechanical description of their delocalized $\pi$ electrons are being developed by several researchers, ${ }^{60-62}$ so a better understanding of specific interactions that could be employed in our solvatochromic MEH-PPV studies should be feasible soon.

C. Implications for Interchain Species in Conjugated Polymers. Even with all the potential uncertainties in the analysis, the opposite signs of the spectral shifts observed in different spatial regions suggest that there are multiple kinds of emissive interchain species with different degrees of charge separation in annealed MEH-PPV films. Despite the difficulties inherent in the choice of the geometric and dielectric parameters defining the model, the calculated values of $\Delta \mu_{\mathrm{e}}$ from eq 2 (listed in Table 2) make reasonable physical sense. Table 2 shows that a small fraction of the interchain species exhibit a large increase of $\sim 9-13 \mathrm{D}$ in dipole moment upon excitation, ${ }^{63}$ while the majority of interchain species show a modest dipole decrease of $\sim 4-7 \mathrm{D}$. While these calculated changes in dipole moment may seem quite large (the separation of a fundamental unit of charge over $1 \AA$ corresponds to a $4 \mathrm{D}$ dipole moment), their magnitude is consistent with changes in dipole measured for MEH-PPV films using electroabsorption (Stark) spectroscopy. ${ }^{64}$ Our calculations of large values of $\Delta \mu_{\mathrm{e}}$ from eq 2 are also consistent with recent molecular dynamics (MD) simulations of simple chromophores at interfaces. ${ }^{65}$ Even if the magnitude of the calculated dipole changes is in error by a factor of 2 or more, the fact that different spatial regions of the film show shifts with opposite sign is strongly indicative of the presence of multiple classes of emissive interchain species.

For the interfacial emission collected from most regions of annealed MEH-PPV films, the solvatochromic blue shift suggests that the typical interchain excited state is predominantly neutral in character. Given that the magnitude of the groundstate dipole for MEH-PPV chains is expected to be around the same size as the calculated values of $\Delta \mu_{\mathrm{e}}$ in Table 2, this implies that the interchain excited state must have a dipole moment that approaches zero, as expected by symmetry: an excited state with a very small dipole moment is expected for interchain species that are neutraly delocalized over multiple chain segments, such as excimers or aggregates. In the few regions where the interfacial emission shows the solvatochromic red shift, the fact that the excited state has a large dipole moment ( 9-13 D larger than that of the ground state) suggests strong excited-state charge separation. Because typical interchain distances in conjugated polymer films are $\sim 4 \AA$, ${ }^{66}$ this magnitude of $\Delta \mu_{\mathrm{e}}$ suggests nearly complete separation of unit charges between adjacent chains if the radius of the emitter $R$ $=5 \AA$, consistent with assignment to interchain polaron pairs. ${ }^{6}$ This same dipole moment could be achieved for larger choices of the radius of the emitter if only fractional charges were separated, which still would be suggestive of interchain species comprised of highly charge-asymmetric exciplexes.

We conclude our discussion by speculating on the reasons that the solvatochromic shifts in annealed MEH-PPV films are spatially inhomogeneous. It is well-known from photoconductivity and other measurements that the presence of defects such as carbonyl groups ${ }^{67}$ in conjugated polymer films can lead to increased separation of charge. Moreover, Lee et al. have demonstrated that chain ends can dramatically increase interchain electronic interactions in annealed polymer films. ${ }^{68}$ In addition, recent work studying the emission from single molecules of PPV derivatives also suggests that chain defects such as cis bonds or tetrahedrally coordinated backbone carbon atoms can alter the way polymer chains fold and hence change the degree of interchain interactions. ${ }^{69,70}$ If any species such as carbonyl groups, chain ends, cis bonds, or tetrahedral sites are present in our MEH-PPV films, the annealing process would allow those defects to freely flow in the polymer melt. Because the entropy of mixing polymer segments with defects into the rest of the polymer film is very low, it is likely that the defects will phase segregate into spatially inhomogeneous regions, presumably the places where we observe the solvatochromic red shift. Taken together, this line of reasoning suggests that neutral excimer-like species are the norm for interchain excitations in conjugated polymer films but that occasional chargeseparated interchain species can be produced if the excitation occurs on a segment near an intrinsic defect such as a carbonyl group or a chain end.

As a first step toward exploring what types of defects might be responsible for the production of the solvatochromically redshifted charge-separated domains, we have performed NSOM interfacial solvatochromism experiments on annealed MEH-PPV films with a lower molecular weight. These experiments allow us to explore the effects of chain ends on the interchain emission, because the low molecular weight $(\sim 60 \mathrm{kDa})$ films have $\sim 8$ times the density of chain ends as the high molecular weight films ( $\sim 450 \mathrm{kDa})$ studied above. Figure 9 compares images constructed by collecting PL through the 700-nm long-pass filter from annealed MEH-PPV films with different molecular weights under EG. Panel a shows the typical solvatochromically redshifted domains observed for our high molecular weight films, (produced in the same manner as Figure 7d above), while panel b shows the results for a the same measurement conducted on a low molecular weight annealed MEH-PPV film. The figure makes it clear that the red-shifting domains increase in size as the molecular weight of the film is decreased: the typical redshifted domains in the low molecular weight films are $\sim 3-6$ $\mu \mathrm{m}$ in diameter. This larger size can be interpreted in one of two ways: either the chain ends themselves contribute to the existence of the solvatochromically red-shifting domains, or the fact that the chains have a lower molecular weight allows more facile phase segregation of other defects to form the red-shifting domains. Either way, the data in Figure 9 strongly support our 


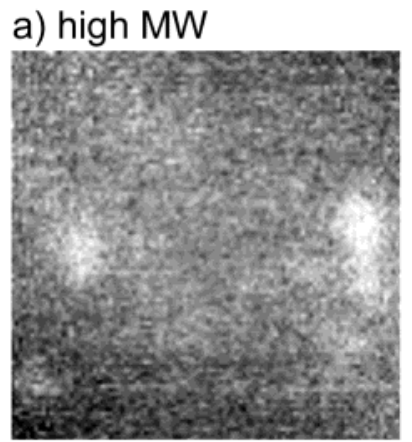

\section{b) low MW}

Rel. PL Intensity 0.65

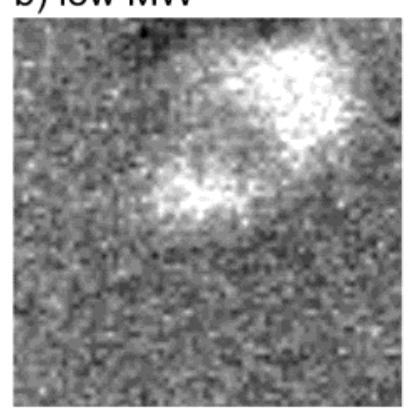

1.00

Figure 9. Solvatochromism of (a) high ( $450 \mathrm{kDa})$ and (b) low molecular weight $(\sim 60 \mathrm{kDa})$ annealed films. NSOM images were produced with the films in contact with EG collecting emission wavelengths $\geq 700 \mathrm{~nm}$ (as in Figure 7d) to show the solvatochromically red-shifted domains (seen as bright features). The domains observed in low molecular weight MEH-PPV films are larger in diameter than those observed in high molecular weight films. The field of view for each image is $(15 \mu \mathrm{m})^{2}$.

conclusion that the defects that catalyze interchain charge separation are intrinsic.

\section{Conclusions}

In this paper, we have shown that it is possible to measure the solvatochromism of solid film samples by using NSOM to selectively collect only the emission from the interface in contact with the applied liquid. We calibrated our interfacial solvatochromism technique by measuring the known dipole change of the laser dye DCM embedded in a PMMA host. We then employed our method with annealed MEH-PPV films and observed a spatial variation in the solvatochromic behavior. The majority of the regions studied exhibit a blue shift of the PL in contact with high-polarity liquids relative to the PL collected with the film in contact only with nitrogen gas. A few small (ca. 1-2 $\mu \mathrm{m}$ diameter) spatially localized areas of the films, however, exhibit a large solvatochromic red shift. Analysis of the data using an interfacial dielectric continuum model indicates that the solvatochromic blue-shifting regions correspond to a localized decrease in dipole moment upon excitation of $\sim 4-7$ $\mathrm{D}$, while the solvatochromic red-shifting regions exhibit a dipole increase on excitation of $\sim 9-13 \mathrm{D}$.

We interpret the observed spatial variation as evidence that several types of interchain species are present in annealed films of MEH-PPV. Independent of any of the numerical values that we calculate for $\Delta \mu_{\mathrm{e}}$, the blue-shifting regions indicate that charge-neutral excimer-like species are the primary contributors to the interchain emission from conjugated polymers, while the red-shifting regions suggest that interchain emission from polaron pairs or exciplexes is also present. The facts that the red-shifting regions are spatially localized and that the size of the regions correlates inversely with molecular weight suggest that charge-separated interchain species are correlated with the presence of intrinsic defects. We note that all of our conclusions apply only to emissive interchain species; we can by no means rule out the presence of nonemissive interchain species that may have a substantially different electronic character. ${ }^{7,8}$ For the emissive interchain species that we do observe, however, the fact that their electronic properties depend so sensitively on the chain packing or the presence of defects or both goes a long way toward explaining the controversy regarding the nature of interchain species in the literature. Because different groups tend

to study polymers from different batches that also have been processed in different ways, it is not surprising that there have been so many different conclusions about the existence and nature of interchain electronic species in conjugated polymer films.

We close this paper by comparing our solvatochromic results to recent work in which we measured the spatial variation of third harmonic generation (THG) efficiency as a function of wavelength in the same annealed MEH-PPV films. ${ }^{71}$ In our THG measurements, we observed numerous small ( $200 \mathrm{~nm}$ diameter) domains that are characterized by a red shift in the thirdharmonic resonance relative to that observed from isolated MEH-PPV chains. This red-shifted resonance is indicative of an absorption feature that is shifted to lower energies than that of a single chain, most likely the signature of a weakly absorbing interchain species. The spatial extent and frequency with which these THG features occur, however, are quite different from those observed in the present solvatochromic study. As discussed in more detail elsewhere, ${ }^{71}$ the two techniques, solvatochromism and THG NSOM, constitute two very different means for probing a conjugated polymer sample. THG NSOM experiments probe the bulk of the films and are most sensitive to groundstate sample properties such as absorption and polarizability, whereas NSOM solvatochromism measures the local environment surrounding emissive excited-state species near the surface of the films. Thus, the two novel techniques provide complementary information about the complex processes underlying the formation and behavior of interchain electronic species in conjugated polymers.

Acknowledgment. This work was supported by the NSF under Grants DMR-9971842 (UCLA), CHE-9981847 (UCSC), and CHE-9727302 (UCB). The UCLA group gratefully acknowledges the support of the Petroleum Research Fund, administered by the American Chemical Society, through Grant number 37029-AC5,7. B.J.S. is a Cottrell Scholar of Research Corporation, an Alfred P. Sloan Foundation Research Fellow, and a Camille Dreyfus Teacher-Scholar. We thank Steve Ruzin and Denise Schichnes in the CNR Biological Imaging Facility at UCB for assistance with the confocal microscopy measurements.

\section{References and Notes}

(1) Friend, R. H.; Gymer, R. W.; Holmes, A. B.; Burroughes, J. H.; Marks, R. N.; Taliani, C.; Bradley, D. D. C.; Dos Santos, D. A.; Brédas, J.-L.; Logdlund, M.; Salaneck, W. R. Nature 1999, 397, 121-128. 1592.

(2) Lovinger, A. J.; Rothberg, L. J. J. Mater. Res. 1996, 11, 1581-

(3) Burroughes, J. H.; Bradley, D. D. C.; Brown, A. R.; Marks, R. N.; Mackay, K.; Friend, R. H.; Burns, P. L.; Holmes, A. B. Nature 1990, 347, $539-541$.

(4) Greenham, N. C.; Samuel, I. D. W.; Hayes, G. R.; Phillips, R. T.; Kessener, Y. A. R. R.; Moratti, S. C.; Holmes, A. B.; Friend, R. H. Chem. Phys. Lett. 1995, 241, 89-96.

(5) Becker, H.; Burns, S. E.; Friend, R. H. Phys. Rev. B 1997, 56 , $1893-1905$

(6) Yan, M.; Rothberg, L. J.; Papadimitrakopoulos, F.; Galvin, M. E.; Miller, T. M. Phys. Rev. Lett. 1994, 72, 1104-1107.

(7) Collison, C. J.; Rothberg, L. J.; Treemaneekarn, V.; Li, Y. Macromolecules 2001, 34, 2346-2352.

(8) Wang, P.; Collison, C. J.; Rothberg, L. J. J. Photochem. Photobiol. A 2001, 144, 63-68.

(9) Nguyen, T.-Q.; Martini, I. B.; Liu, J.; Schwartz, B. J. J. Phys. Chem. B 2000, 104, 237-255.

(10) Nguyen, T.-Q.; Kwong, R. C.; Thompson, M. E.; Schwartz, B. J. Appl. Phys. Lett. 2000, 76, 2454-2456.

(11) Lupton, J. M.; Samuel, I. D. W.; Beavington, R.; Frampton, M. J.; Burn, P. L.; Bassler, H. Phys. Rev. B 2001, 63, 155206/1-8.

(12) Jenekhe, S. A.; Osaheni, J. A. Science 1994, 265, 765-768. 
(13) Samuel, I. D. W.; Rumbles, G.; Collison, C. J.; Friend, R. H.; Moratti, S. C.; Holmes, A. B. Synth. Met. 1997, 84, 497-500.

(14) Jakubiak, R.; Rothberg, L. J.; Wan, W.; Hsieh, B. R. Synth. Met. 1999, 101, 230-233.

(15) Nguyen, T.-Q.; Doan, V.; Schwartz, B. J. J. Chem. Phys. 1999, 110, 4068-4078.

(16) Blatchford, J. W.; Gustafson, T. L.; Epstein, A. J.; Vandenbout, D. A.; Kerimo, J.; Higgins, D. A.; Barbara, P. F.; Fu, D. K.; Swager, T. M.; Macdiarmid, A. G. Phys. Rev. B 1996, 54, R3683-R3686.

(17) Blatchford, J. W.; Jessen, S. W.; Lin, L. B.; Gustafson, T. L.; Fu, D. K.; Wang, H. L.; Swager, T. M.; Macdiarmid, A. G.; Epstein, A. J. Phys. Rev. B 1996, 54, 9180-9189.

(18) Lemmer, U.; Heun, S.; Mahrt, R. F.; Scherf, U.; Hopmeier, M.; Siegner, U.; Gobel, E. O.; Mullen, K.; Bassler, H. Chem. Phys. Lett. 1995, 240, 373-378.

(19) Pauck, T.; Hennig, R.; Perner, M.; Lemmer, U.; Siegner, U.; Mahrt, R. F.; Scherf, U.; Mullen, K.; Bassler, H.; Gobel, E. O. Chem. Phys. Lett. 1995, 244, 171-176.

(20) Mahrt, R. F.; Pauck, T.; Lemmer, U.; Siegner, U.; Hopmeier, M.; Hennig, R.; Bassler, H.; Gobel, E. O.; Bolivar, P. H.; Wegmann, G.; Kurz, H.; Scherf, U.; Mullen, K. Phys. Rev. B 1996, 54, 1759-1765.

(21) Yan, M.; Rothberg, L. J.; Kwock, E. W.; Miller, T. M. Phys. Rev. Lett. 1995, 75, 1992-1995.

(22) Kraabel, B.; Klimov, V. I.; Kohlman, R.; Xu, S.; Wang, H. L.; McBranch, D. W. Phys. Rev. B 2000, 61, 8501-8515.

(23) Beljonne, D.; Cornil, J.; Sirringhaus, H.; Brown, P. J.; Shkunov,

M.; Friend, R. H.; Bredas, J. L. Adv. Funct. Mater. 2001, 11, 229-234.

(24) Nguyen, T.-Q.; Yee, R. Y.; Schwartz, B. J. J. Photochem. Photobiol. A 2001, 144, 21-30.

(25) Yang, C. Y.; Hide, F.; DiazGarcia, M. A.; Heeger, A. J.; Cao, Y. Polymer 1998, 39, 2299-2304.

(26) Nguyen, T.-Q.; Wu, J.; Doan, V.; Schwartz, B. J.; Tolbert, S. H. Science 2000, 288, 652-656.

(27) Conwell, E. M. Phys. Rev. B 1998, 57, 14200-14202.

(28) Grell, M.; Bradley, D. D. C.; Long, X.; Chamberlain, T.; Inbaseka-

ran, M.; Woo, E. P.; Soliman, M. Acta Polym. 1998, 49, 439-444.

(29) Reichardt, C. Solvent effects in organic chemistry; Verlag Chemie: Weinheim, Germany, 1979.

(30) For many systems, solvatochromic behavior also can be observed in absorption.

(31) If one takes into account the different directions of the groundand excited-state dipoles, then the shift goes as the dot product of the groundstate dipole vector with the vector difference between the excited and ground states. For more details, see: Liptay, W. Z. Naturforsch. 1965, 20a, 14411471.

(32) Lippert, E. Z. Naturforsch. 1955, 10a, 541-546.

(33) Mataga, N.; Kaifu, Y.; Koizumi, M. Bull. Chem. Soc. Jpn. 1956, $29,465-470$.

(34) McRae, E. G. J. Phys. Chem. 1957, 61, 562-572

(35) Greenfield, S. R.; Svec, W. A.; Gosztola, D.; Wasielewski, M. R. J. Am. Chem. Soc. 1996, 118, 6767-6777.

(36) Paesler, M. A.; Moyer, P. J. Near-field optics: theory, instrumentation, and applications; Wiley: New York, 1996.

(37) Jackson, J. D. Classical electrodynamics, 3rd ed.; Wiley: New York, 1999.

(38) Schaller, R. D.; Johnson, J. C.; Wilson, K. R.; Lee, L. F.; Haber, L. H.; Saykally, R. J. J. Phys. Chem. B 2002, 106, 5143-5154.

(39) Enderle, T.; Ha, T.; Chemla, D. S.; Weiss, S. Ultramicroscopy 1998, 71, 303-309. 1609.
(41) Zhou, S. Q.; Wu, C. Macromolecules 1996, 29, 4998-5001.

(42) Drake, P. A.; Bohn, P. W. Anal. Chem. 1995, 67, 1766-1771.

(43) Benjamin, I. J. Phys. Chem. A 1998, 102, 9500-9506.

(44) Nguyen, T.-Q.; Schwartz, B. J.; Schaller, R. D.; Johnson, J. C.; Lee, L. F.; Haber, L. H.; Saykally, R. J. J. Phys. Chem. B 2001, 105, $5153-$ 5160 .

(45) Schaller, R. D.; Roth, C.; Raulet, D. H.; Saykally, R. J. J. Phys. Chem. B 2000, 104, 5217-5220.

(46) Lee, L. F.; Schaller, R. D.; Haber, L. H.; Saykally, R. J. Anal. Chem 2001, 73, 5015-5019.

(47) Meyer, M.; Mialocq, J. C.; Perly, B. J. Phys. Chem. 1990, 94, 98104.

(48) Meyer, M.; Mialocq, J. C. Opt. Commun. 1987, 64, 264-268.

(49) Calculations using values of $a$ set equal to $R / 4$ produced nonphysically large values for the change in dipole moment.

(50) Bosshard, C.; Knopfle, G.; Pretre, P.; Gunter, P. J. Appl. Phys. 1992, 71, 1594-1605. 9387. (52) Stevenson, R.; Granstrom, M.; Richards, D. Appl. Phys. Lett. 1999, $75,1574-1576$

(53) Webster, S.; Smith, D. A.; Batchelder, D. N.; Lidzey, D. G.; Bradley, D. D. C. Ultramicroscopy 1998, 71, 275-279.

(54) Hsu, J. H.; Wei, P. K.; Fann, W. S.; Chuang, K. R.; Chen, S. A. Ultramicroscopy 1998, 71, 263-267.

(55) DeAro, J. A.; Weston, K. D.; Buratto, S. K.; Lemmer, U. Chem Phys. Lett. 1997, 277, 532-538.

(56) The interchain coupling of neighboring chromophores typically leads to a splitting of the ground and excited states, as described in: Cornil, J.; Beljonne, D.; dos Santos, D. A.; Calbert, Ph.; Brédas, J.-L. Thin Solid Films 2000, 363, 72-75. For the lowest-energy excited excimer state, we would expect the dipole moment to be near zero by symmetry.

(57) Hide, F.; Schwartz, B. J.; DiazGarcia, M. A.; Heeger, A. J. Synth. Met. 1997, 91, 35-40.

(58) Boudrioua, A.; Hobson, P. A.; Matterson, B.; Samuel, I. D. W.; Barnes, W. L. Synth. Met. 2000, 111, 545-547.

(59) Zhang, X. Y.; Walker, R. A. Langmuir 2001, 17, 4486-4489.

(60) Kobrak, M. N.; Bittner, E. R. Phys. Rev. B 2000, 62, 11473-11486.

(61) Moore, E. E.; Yaron, D. J. Chem. Phys. 1998, 109, 6147-6156 34

(63) We note that the parameters for the peak position and width of the reddest SRPL band centered near $730 \mathrm{~nm}$ were relatively insensitive to the details of the fit because of the band's low amplitude.

(64) Peteanu, L. A., manuscript in preparation.

(65) Vieceli, J.; Benjamin, I., manuscript in preparation.

(66) Cornil, J.; dos Santos, D. A.; Crispin, X.; Silbey, R.; Brédas, J.-L. J. Am. Chem. Soc. 1998, 120, 1289-1299.

(67) Antoniadis, H.; Rothberg, L. J.; Papadimitrakopoulos, F.; Yan, M. Galvin, M. E.; Abkowitz, M. A. Phys. Rev. B 1994, 50, 14911-14915.

(68) Lee, J. I.; Klaemer, G.; Miller, R. D. Chem. Mater. 1999, 11, $1083-$ 1088 .

(69) Hu, D. H.; Yu, J.; Wong, K.; Bagchi, B.; Rossky, P. J.; Barbara, P. F. Nature 2000, 405, 1030-1033.

(70) Claudio, G. C.; Bittner, E. R. J. Chem. Phys. 2001, 115, 95859593.

(71) Schaller, R. D.; Snee, P. T.; Johnson, J. C.; Lee, L. F.; Wilson, K R.; Haber, L. H.; Saykally, R. J.; Nguyen, T.-Q.; Schwartz, B. J. J. Chem. Phys., in press.

(72) Brandrup, J.; Immergut, E. H. Polymer handbook, 3rd ed.; Wiley: New York, 1989. 